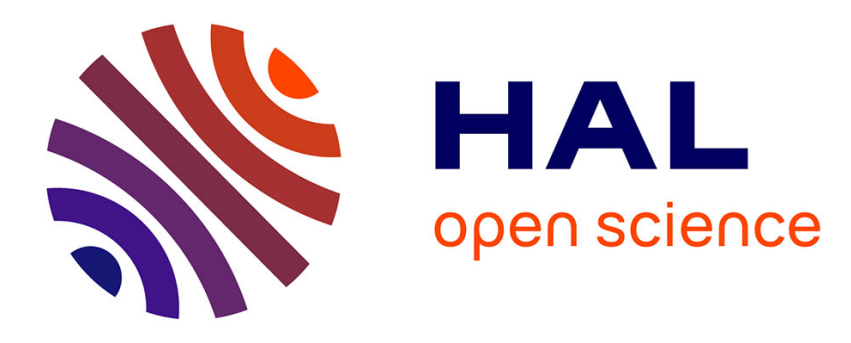

\title{
Measurement of Dislocation Tunneling Through a Pinning Atom
}

T. Kosugi, D. Mckay, A. Granato

\section{To cite this version:}

T. Kosugi, D. Mckay, A. Granato. Measurement of Dislocation Tunneling Through a Pinning Atom. Journal de Physique IV Proceedings, 1996, 06 (C8), pp.C8-863-C8-866. 10.1051/jp4:19968186 jpa00254623

\section{HAL Id: jpa-00254623 https://hal.science/jpa-00254623}

Submitted on 1 Jan 1996

HAL is a multi-disciplinary open access archive for the deposit and dissemination of scientific research documents, whether they are published or not. The documents may come from teaching and research institutions in France or abroad, or from public or private research centers.
L'archive ouverte pluridisciplinaire HAL, est destinée au dépôt et à la diffusion de documents scientifiques de niveau recherche, publiés ou non, émanant des établissements d'enseignement et de recherche français ou étrangers, des laboratoires publics ou privés. 
JOURNAL DE PHYSIQUE IV

Colloque C8, supplément au Journal de Physique III, Volume 6, décembre 1996

\title{
Measurement of Dislocation Tunneling Through a Pinning Atom
}

\author{
T. Kosugi, D.McKay* and A.V. Granato* \\ Department of Physics, Hiroshima University, Higashi-Hiroshima 739, Japan \\ * Department of Physics, University of Illinois at Urbana-Champaign, Urbana, Illinois 61801, U.S.A.
}

\begin{abstract}
We find convincing evidence of dislocation tunneling in the superconducting state of an aluminum crystal below $1 \mathrm{~K}$, that is, a flattening of the temperature dependence of applied stress amplitude between $0.1-0.5 \mathrm{~K}$ for constant decrement of amplitude dependent internal friction (ADIF). Since the interaction potential of a dislocation with an impurity atom has been measured quantitatively by the ADIF, the height $\Delta U$ and width $\Delta x$ of the potential barrier is given as a function of applied stress. For the crossover temperature $T^{*}$ between quantum tunneling and classical thermal activation given as $T^{*}=(h / k) \sqrt{\Delta U} /(2 \sqrt{M} \Delta x)$, thus the only adjusting parameter is an effective mass $(M)$ of the dislocation segment that participates in the tunneling process. Using a modified Cottrell potential which has been determined as the interaction potential, $\mathrm{T}^{*}$ is predicted to be $0.7 \mathrm{~K}$ when $\mathrm{M}$ is taken to be $100 \mathrm{Al}$ atomic masses. This magnitude is consistent with the experimental results.
\end{abstract}

\section{INTRODUCTION}

Although quantum mechanics has no inconsistency with observations in any phenomena, it is generally difficult to explain macroscopic system behavior directly from the fundamental equations of quantum mechanics. For instance, although the probability of quantum tunneling of a particle of mass $M$ through a potential barrier of height $\Delta \mathrm{U}$ and width $\Delta \mathrm{x}$ is given by $\mathrm{P}=v_{0} \exp \left(-2 \Delta \mathrm{x} \sqrt{2 \mathrm{M} \Delta \mathrm{U} / \mathrm{h}^{2}}\right)$ where $v_{0}$ is an effective attack frequency, tunneling in a macroscopic system with many degrees of freedom and interacting with an atmosphere such as electrons and phonons is an unsolved problem of current interest because this issue could give us critical conditions for discriminating between quantum mechanical behavior and classical behavior in macrosystems.

Recently, macroscopic quantum tunneling (MQT) has been considered in systems where the tunneling entity is linear rather than point-like. Examples include phase-slip vortices in superfluid ${ }^{4} \mathrm{He}$ [1], quantum creep of magnetic flux lines in superconductors[2], and vortex creep in neutron stars[3]. A dislocation in a crystal is another macroscopic system with distinct characteristics which make it a good choice for observing MOT and may give it an advantage over some of the others[4,5]. For instance, the thermal activation process of a dislocation overcoming elementary barriers has been well studied[6]. There have been many dislocation tunneling studies in experiments $[4,7,8]$ such as creep, plastic flow-stress and internal friction measurements, and in theoretical investigations $[4,9,10]$ for a point-like barrier such as a forest dislocation or an impurity atom, and Peierls potential barrier. The dislocation system for tunneling, however, is not specified enough for macroscopic deformation measurements except for the large Peierls-barrier case. Theoretical studies presently predict considerably diverse magnitudes for the crossover temperature below which classical thermal activation gives way to quantum tunneling or some other quantum effect[4], from below $1 \mathrm{~K}$ to several tens of $\mathrm{K}$, because of rather rough approximations and unspecified parameter values. Thus a specified system with specific parameters is needed to have a more quantitative understanding.

It has been clear recently that a quantitative approach for dislocation-barrier interaction is possible with amplitude-dependent internal friction (ADIF) measurements[6]. In particular, low-temperature ADIF would provide detailed information on the dislocation-impurity atom interaction by using a quantitative breakaway string model of a pinned dislocation (Granato-Lucke model) for the temperature dependence of the stress amplitude for constant ADIF[11]. ADIF is also nondestructive and repeatable for the same sample, so more accurate information will be obtained than from plastic deformation measurements. For the region of classical behavior of thermally activated breakaway, the $T^{2 / 3}$ dependence of stress amplitude on temperature in the low temperature region is established for the dislocation-single pinning point interaction[12]. Furthermore, for aluminum crystals, the detailed form of the interaction potential has been determined from 
a wide range of temperature dependence of ADIF, so the height $\Delta U$ and width $\Delta x$ of the potential barrier is well specified as the function of applied stress $[6,8]$.

\section{PRINCIPLE OF MEASUREMENT}

The system we choose for the dislocation-tunneling search is a dislocation pinned by impurity atoms. Since the longest double loop in the dislocation segment controls the breakaway, we consider the pinning atom in the double loop (Fig.1). At a stress $\sigma$ close to the mechanical breakaway stress $\sigma_{M}$, since the interaction force between a dislocation and a pinning atom is well approximated as a parabola, $\Delta \mathrm{U}$ of the effective potential is given as $\Delta \mathrm{U}=\mathrm{U}_{0}\left(1-\mathrm{\sigma} / \mathrm{\sigma}_{\mathrm{M}}\right)^{3 / 2}$. On the other hand, the rate of breakaway is given as $v_{\mathrm{th}}=v_{0} \exp (-\Delta \mathrm{U} / \mathrm{kT})$ for thermal activation and $v_{t u}=v_{0} \exp \left(-2 \sqrt{2} \alpha M^{1 / 2}(\Delta U)^{1 / 2} \Delta x / \hbar\right)$ for tunneling, respectively. Here $\alpha$ is a numerical factor $(\approx 0.7)$ and $M$ is an effective mass of the dislocation segment which participates in tunneling. If the measurement is made for constant flowstrain-rate or constant ADIF, $\triangle \mathrm{U} / \mathrm{kT}$ is almost constant, but the relative rate of tunneling increases exponentially with decreasing temperature. Thus there should be crossover between quantum tunneling and classical thermal activation processes. The crossover temperature $T^{*}$ may be defined as $T^{*}=\hbar(\Delta U)^{1 / 2} /\left(2 \alpha \mathrm{k} \Delta x(2 \mathrm{M})^{1 / 2}\right)$, equating the exponent of the two rates. We consider two different interaction potentials for the dislocation-pinning atom interaction. One is the Cottrell type based on continuum-elasticity theory and the other is a modified Cottrell $[6,8]$ determined from the thermal activation analysis of the ADIF measurement in Al. The distinct difference is that the modified Cottrell has a rather narrow force width compared with the Cottrell because of a different core structure, i.e., narrower by 2.8 times for the same $\sigma / \sigma_{M}$ in the high stress region. So the tunneling probability is expected to be higher for the modified Cottrell than for the Cottrell. If $M$ is taken to be a dislocation mass of 100 atomic lengths, the crossover temperature is predicted to be $0.78 \mathrm{~K}$ and $0.62 \mathrm{~K}$ for a $\mathrm{Mg}$ atom (binding energy $0.19 \mathrm{eV}$ ) and a $\mathrm{Zn}$ atom $(0.11 \mathrm{eV})$ in $\mathrm{Al}$, respectively $[8]$.

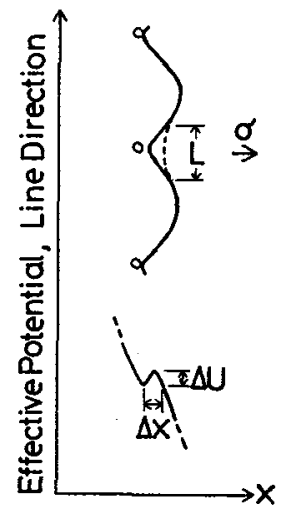

Fig.1

Double loop of pinned dislocation with applied stress and effective potential barrier.

\section{EXPERIMENTAL}

A zone-refined aluminum single crystal was chosen for the sample for the following reasons. First, an FCC crystal has a very small Peierls stress $\left(-10^{-5} \mu\right.$, $\mu$ : shear modulus), so the specified interaction between a dislocation and a point defect can be studied. Second, the details of the interaction potential of a dislocation with a impurity atom have been well studied. Third, very pure crystals are available, i.e., the total impurity is about $1 \mathrm{ppm}$. Thus the stress amplitude where ADIF starts is very small and it is very important to reduce the heat generated from internal friction for low-temperature measurements below $1 \mathrm{~K}$. For ADIF a composite oscillator method using $-18.5^{\circ} \mathrm{X}$-cut quartz rod was used[13]. For temperature control a dilution refrigerator was used from $0.1 \mathrm{~K}$ to $5 \mathrm{~K}$.

The amplitude dependence of the decrement of the Al sample at $0.15 \mathrm{~K}$ is shown in Fig.2. The temperature in the sample chamber can be monitored either by directly attaching a Ge resistance thermometer
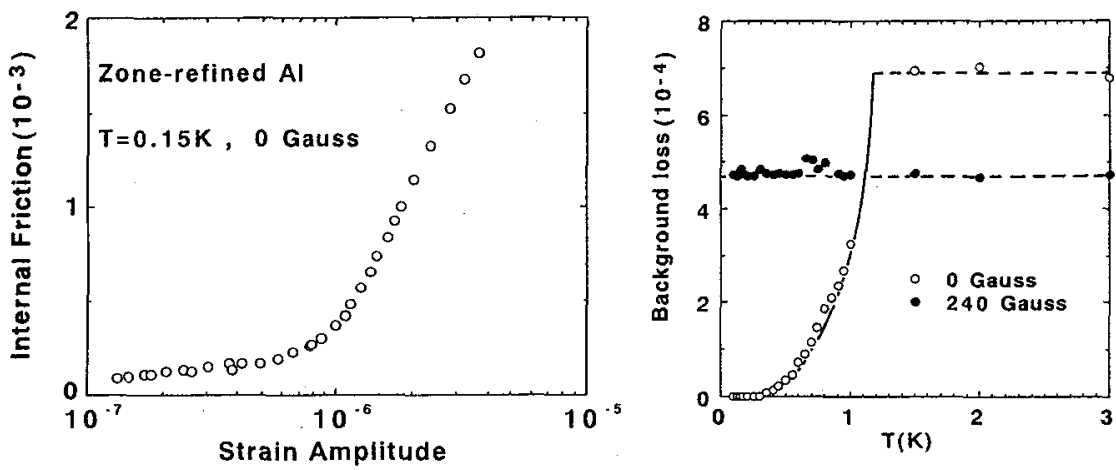

Fig.2 (left)

Internal friction vs strain amplitude at $0.15 \mathrm{~K}$.

Fig.3 (right) Amplitudeindependent internal friction vs temperature. Solid line is BCS theory curve. 
to the center of the sample or by positioning it on an aluminum dummy rod nearby with a small amount of ${ }^{3} \mathrm{He}$ exchange gas $(0.1 \mathrm{tom})$ introduced into the chamber to provide thermal contact between sample, thermometer and cooling pot. The indirect method is preferred so that the resonance of the sample is not disturbed, and a comparison between the two indicates that the indirect method provides an accuracy of about $0.01 \mathrm{~K}$ down to $0.15 \mathrm{~K}$. Below this temperature, the low vapor pressure of the exchange gas and heating of the sample due to the internal dissipation make temperature control more difficult.

The background loss $\Delta_{\mathrm{I}}$ (amplitude-independent internal friction) is plotted in Fig.3. For zero magnetic field, the temperature dependence below Tc follows BCS theory, and the magnitude of the decrement above $\mathrm{Tc}$ is consistent with the high sample purity, i.e., the resistivity ratio for RT to $4.2 \mathrm{~K}$ is estimated to be 15000 from the decrement[14]. Furthermore the decrement $\Delta_{\mathrm{I}}$ of the normal state with a magnetic field of $240 \mathrm{G}$, which is about $2 / 3$ of that with zero field, is explained by the increase of electrical resistivity due to magnetoresistance effects[15]. Thus the background loss is mainly due to acoustic wave-conduction electron interaction. Next after subtracting the background loss from the decrement-strain amplitude curves for each temperature, the strain amplitude necessary for keeping a constant ADIF $(\Delta \mathrm{H})$ is determined and plotted in Fig.4 for the superconducting (s) state and normal (n) state. Unfortunately the normal state data are not good enough to discuss precisely because of the lack of higher temperature data above Tc and relatively large fluctuations of the data. Thus we will mainly discuss the s state data in section 4 .

\section{DISCUSSION}

The negative temperature dependence above $T c$ is due to a thermal activation process and follows the $\mathrm{T}^{2 / 3}$ law as predicted. From the slope, the binding energy is estimated to be about 0.09 to $0.13 \mathrm{eV}$ for lower to higher $\operatorname{ADIF}\left(\Delta_{\mathrm{H}}\right)$ level assuming the modified Cottrell potential[6,8]. This is a reasonable magnitude for the interaction. Below $\mathrm{Tc}$, there is a rapid decrease of strain amplitude with decreasing temperature in the s state. This superconducting effect is known to be universal, appearing in other metals such as $\mathrm{Pb}, \mathrm{Sn}$ and $\mathrm{In}[4]$. The origin is generally explained by the inertial effects due to the decrease of electron scattering against dislocation motion[16]. The damping constant $\mathrm{B}_{\mathrm{e}}$ due to electron scattering is considered to decrease as the acoustic damping due to conduction electrons in BCS theory and is predicted to be negligible (less than 3 $\%$ ) around $0.4 \mathrm{Tc}$. Thus the decrease of strain amplitude will end around $0.5 \mathrm{~K}$ (about $0.6 \mathrm{~K}^{2 / 3}$ ), and then an increase of strain amplitude with decreasing temperature is expected if a thermal activation process still controls the breakaway. In fact in the case of $\mathrm{Pb}$ crystals, there is the same negative slope of strain amplitude below $3 \mathrm{~K}$ (about $0.4 \mathrm{Tc}$ for $\mathrm{Pb}$ ) in the $\mathrm{s}$ and $\mathrm{n}$ state[17]. There is, however, no increase of strain amplitude in the Al sample down to $0.15 \mathrm{~K}$, but a flattening instead between 0.15 and $0.5 \mathrm{~K}$. We may conclude that this flattening is due to a tunneling process for dislocation breakaway through a pinning atom. The crossover temperature, $\mathrm{T}^{*}$ is estimated to be $0.7 \mathrm{~K}$ for the interaction energy of $0.10 \mathrm{eV}$, by assuming $100 \mathrm{Al}$ atom masses as the effective mass for the barrier of modified Cottrell potential. Thus this conclusion is not unreasonable.

On the other hand it is difficult to see quantum tunneling for the $\mathrm{n}$ state because of scatter in the data so far available. We would like, however, to discuss briefly the expected effect of dissipation on tunneling.

Fig.4

Temperature dependence of strain amplitude required for constant ADIF in

superconducting state (left ; 0 Gauss) and normal state (right; 240 Gauss). Solid lines show $T^{2 / 3}$ dependence. Dashed lines are eye guides.
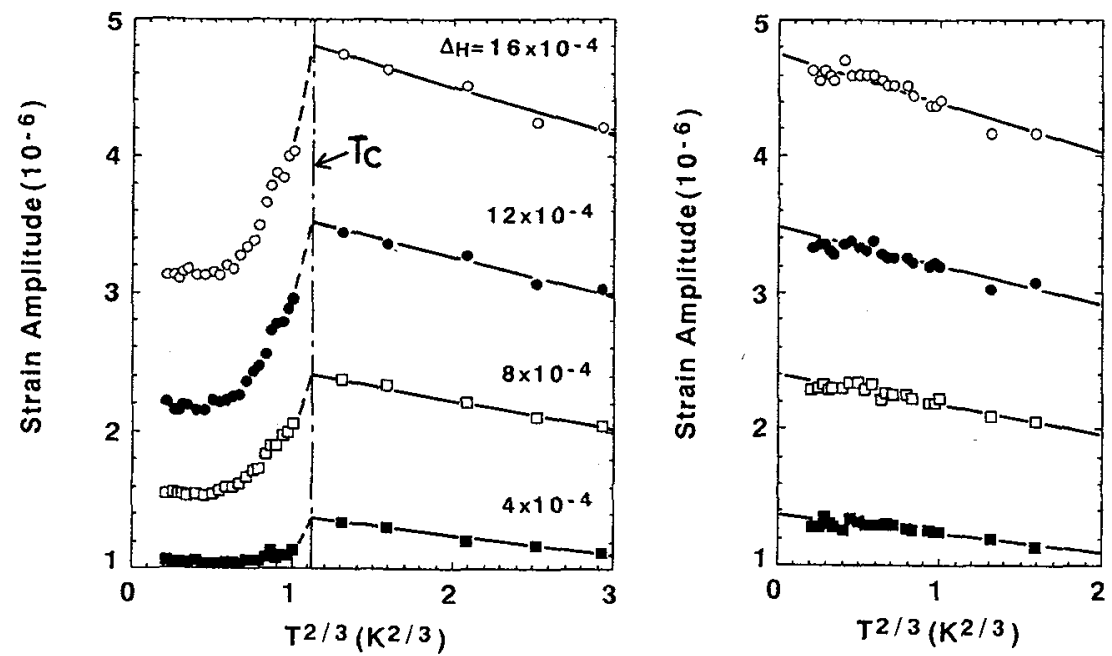
According to the Caldeira-Leggett theory the probability of tunneling becomes smaller by $\exp \left(-\operatorname{An}(\Delta x)^{2} / \hbar\right)$ due to dissipation[18], where $A$ is a numerical factor of order unity and $\eta$ represents a viscosity coefficient. In the case of a dislocation, $\eta$ is given by $B L$, where $B$ is the damping constant and $\mathrm{L}$ is the effective segment participating in the tunneling process. Fig.5 shows a schematic relation of the temperature dependence of stress amplitude for constant ADIF. For temperatures below $0.4 \mathrm{Tc}$, the stress amplitude should increase again with decreasing temperature if thermal activation controls the breakaway. In the data of $\mathrm{Pb}$ crystals down to $1.6 \mathrm{~K}$, this is realized. If the temperature is lowered further below $1 \mathrm{~K}$, the tunneling process will be effective and a flattening of the temperature dependence will be found. After Caldeira-Leggett, the crossover temperature $T^{*}$ is expected to be lower for the $n$ state than the $s$ state due to the difference of electron viscosity. For the $n$ state of $\mathrm{Al}$, where $B$ is $1.4 \times 10^{-6} \mathrm{~Pa} \cdot \mathrm{s}$ from an experiment [19], $\mathrm{L}$ is assumed to be $100 \mathrm{~b}=2.86 \times 10^{-8} \mathrm{~m}$, and $\Delta \mathrm{x}$ is about $2 \times 10^{-11} \mathrm{~m}$, the

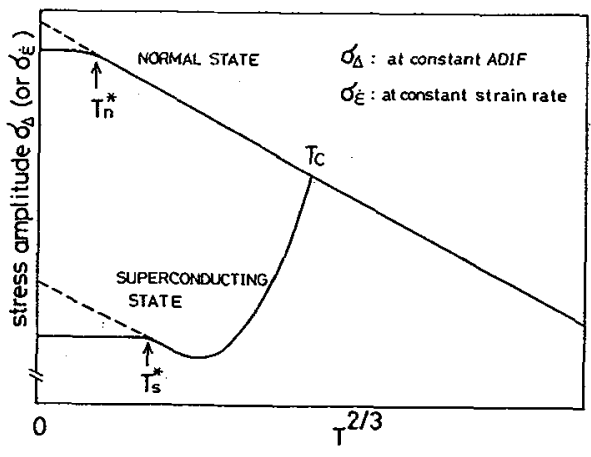

Fig.5

Expected behavior of stress amplitude change with temperature in normal state and superconducting state. exponent $A \eta(\Delta x)^{2} / \hbar$ is estimated to be 0.2 . This is relatively small compared to the exponent $\Delta \mathrm{U} / \mathrm{kT}$ (about a order of ten in our case). So it is expected that the crossover temperature will change by several \% between the $n$ and $\mathrm{s}$ states. From the n-state data shown here it is difficult to say where the sign for tunneling is. We need more measurements to confirm it.

Acknowledgment

This work was supported by the National Science Foundation Grants DMR 90-08469 and 93-19773.

\section{References}

[1]For instance,Davis J.C.,Steinhauser J.,Schwab K., Mukharsky Yu.M.,Sasaki Y.,Packard R.E., Phys. Rev. Lett. 69 (1992) 323.; Ihas G.G.,Avenel O.,Arts R.,Salmekin R.,Phys. Rev. Lett. 69 (1993) 327.

[2]For instance, Gaber M.W., Achar B.N., Phys. Rev. B52 (1995) 1314.; Ephron D., Yazdani A., Kapitulnik A., Beasley M.R., Phys. Rev. Lett. 76 (1996) 1529.

[3]For instance, Link B.K., Esptein R., Astrophys. J. 373 (1991) 592.; Baym G., Epstein R.I., Link B., Physica B178 (1992) 1.; Link B., Epstein R.I., Baym G., Astrophys. J. 403 (1993) 285.

[4]For instance, Startsev V.I., in Dislocations in Solids, Nabarro F.R.N., Ed., Vol.6 (North-Holland, Amsterdam,1983) p.145.

[5]Granato A.V., Proc. ICIFUAS-9, Ke T.S., Ed., (IAP, Beijing, 1990) p. 3.

[6]Kosugi T., Kino T., Mater. Sci. Eng. A164 (1993) 316.

[7]See Dislocations in Solids, Suzuki H., Ninomiya T., Sumino K., Takeuchi S., Eds. (Univ. Tokyo, Tokyo, 1985).

[8]Kosugi T., Kino T., Jpn. J. Appl. Phys. 26 (1987) suppl. 26-3, p881.

[9]Suzuki H., in Mechanical Properties of BCC Metals, Meshii M., Ed., (AIME, New York, 1982) p41.

[10]Petukhov B.V., Sukharev V.Ya., Sov. Low Temp. Phys. 9 (1983) 264.

[11] Granato A.V., Lucke K., J. Appl. Phys. 27 (1956) 583; 52 (1981) 7136.

[12]Schwarz R.B., Granato A.V., Phys. Rev Lett. 34 (1975) 1174.

[13]Schwarz R.B., Rev. Sci. Instrum. 48 (1977) 111.; Kosugi T., Jpn. J. Appl. Phys. 33 (1994) 2862.

[14]Lax E., Phys. Rev. 115 (1959) 1591.

[15]Ueda Y., Kino T., Proc. Int. Conf. Point Defects and Defect Interactions in Metals, Takamura J., Doyama M., Kiritani M., Eds., (Univ. Tokyo Press, Tokyo, 1982) p.294.

[16]Granato A.V., Phys. Rev. Lett. 27 (1971) 660.; Suenaga M., Galligan J.M., Scr. Metall. 5 (1971) 63.

[17]Isaac R.D., Schwarz R.B., Granato A.V., Phys. Rev. B18 (1978) 4143.

[18]Caldeira A.O., Leggett A.J., Phys. Rev. Lett. 46 (1981) 211.

[19]Hikata A., Johnson R.A., Elbaum C., Phys. Rev., B2 (1970) 4856. 